\title{
Review of radiological classifications of pancreatic cancer with peripancreatic vessel invasion: are new grading criteria required?
}

\author{
Y. N. Shen ${ }^{1,2}$, X. L. Bai ${ }^{1,2}$, G. G. Li $i^{1,2}$ and T. B. Liang ${ }^{1,2^{*}}$
}

\begin{abstract}
Pancreatic cancer is mainly diagnosed at an advanced stage when adjacent vessel invasion is present; however, radical resection is potentially curative for selected patients with adjacent vessel invasion. Therefore, accurately judging the resectability of patients with adjacent vessel invasion represents a crucially important step in diagnosis and treatment. Currently, decisions regarding resectability are based on imaging studies, commonly contrast computed tomography (CT). Several radiological classifications have been published for vascular infiltration in pancreatic cancer. However, radiologists always formulate these $\mathrm{CT}$ grading systems according to their own experience, resulting in different judgment methods and parameters. And it is controversial in evaluating performance and clinical application. Besides, the conventional CT grading systems mainly focus on the evaluation of vessel invasion so as to less on the outcome of patient evaluation. In this review, we summarize the mainstream $C T$ grading systems for vascular invasion in pancreatic cancer, with the aim of improving the clinical value of CT grading systems for predicting resectability and survival.
\end{abstract}

Keywords: Pancreatic cancer, Vessel invasion, Computed tomography criterion, Resectability, Review

\section{Background}

Pancreatic cancer is a highly lethal disease with high morbidity and a dismal prognosis $[1,2]$. The 5 -years survival rates for white and black American patients with pancreatic cancer are 8 and 7\%, respectively, and the overall survival rate for all races is only $8 \%$ [2]. Approximately $90 \%$ of patients diagnosed with pancreatic cancer ultimately die of the disease [3]. Patients who do not have specific symptoms in the early stages are frequently diagnosed at an advanced stage, for which surgical therapy is usually not possible. Only $20 \%$ of patients with pancreatic cancer are eligible for one-stage resection [4]; however, $14-30 \%$ of these cases will be found to be unsuitable for resection during surgery [5]. Therefore, the ability to accurately judge the resectability of pancreatic cancer represents a

\footnotetext{
* Correspondence: liangtingbo@zju.edu.cn

${ }^{1}$ Department of Hepatobiliary and Pancreatic Surgery, Second Affiliated Hospital of Zhejiang University School of Medicine, Zhejiang University, Jiefang Road, Shangcheng District, Hangzhou, China

${ }^{2}$ Zhejiang Provincial Key Laboratory of Pancreatic Disease, Hangzhou, China
}

crucially important step in diagnosis and treatment, and could help to more accurately determine appropriate therapeutic approaches and predict the prognosis of individual patients. Moreover, once a patient is confirmed as unsuitable for surgery, palliative or neoadjuvant radiochemotherapy can be given in a timelier manner.

Computed tomography (CT) currently plays an important role in the diagnosis and stage evaluation of pancreatic cancer [6]. Preoperative CT evaluation of peripancreatic vascular infiltration in pancreatic cancer is an essential parameter used to assess whether resection can be performed. Several researchers [7-13] have assessed vascular involvement in pancreatic cancer and established a series of preoperative CT criteria to enable more accurate and reliable assessment. However, differences in imaging practices and interpretation [6], local experience and even the ethnicity of the patients have contributed to variations in these criteria, which are also limited by the technology and resources available. The clinical application of these criteria is also affected by their low accuracy. Therefore, it is imperative to 
Table 1 Loyer's Criteria [7]

\begin{tabular}{ll}
\hline Type & Imaging features \\
\hline A & $\begin{array}{l}\text { Fat plane separates tumor and/or normal } \\
\text { pancreatic parenchyma from adjacent vessels. }\end{array}$ \\
B & $\begin{array}{l}\text { Normal parenchyma separates hypodense tumor } \\
\text { from adjacent vessels. }\end{array}$ \\
C & $\begin{array}{l}\text { Hypodense tumor is inseparable from adjacent } \\
\text { vessels, points of contact form a convexity against vessels. }\end{array}$ \\
D & $\begin{array}{l}\text { Hypodense tumor is inseparable from adjacent vessels, } \\
\text { points of contact form a concavity against or partially } \\
\text { encircle vessels. }\end{array}$ \\
E & $\begin{array}{l}\text { Hypodense tumor encircles adjacent vessels, no fat plane } \\
\text { is identifiable between tumor and vessels. }\end{array}$ \\
F & Tumor occludes vessels. \\
\hline
\end{tabular}

establish widely-accepted criteria for vascular involvement in pancreatic cancer with higher precision and clinical value. Though the National Comprehensive Cancer Network (NCCN) established definitions for borderline resectable pancreatic cancer in 2014 [14] in which imaging features provide an important reference, we hold the opinion that the problems described above still persist. This review aimed to systematically summarize the mainstream CT criteria for peripancreatic vascular infiltration in pancreatic cancer published in recent few decades to provide a more comprehensive reference for radiologists and surgeons. Moreover, this information could contribute to the design and establishment of improved CT imaging criteria for vascular involvement in pancreatic cancer.

\section{Characteristics of existing criteria for vascular involvement in pancreatic cancer Loyer's criteria (1996)}

Loyer et al. [7] suggested CT criteria for vascular infiltration in pancreatic carcinoma in 1996 (Table 1). These criteria could be divided into six types (Type A - F) [7]: in Type A, a fat plane separates the tumor from adjacent vessels; Type
B: normal pancreatic parenchyma separates the tumor from adjacent vessels; Type C: hypodense tumor not separated from vessels, and the points of contact form a convexity against the vessels; Type D: hypodense tumor not separated from vessels, and the points of contact form a concavity against or partially encircle the vessels; Type E: hypodense tumor encircled by adjacent vessels, while the fat plane between the tumor and blood vessels cannot be identified; and Type F: a tumor occluding the vessel (Fig. 1).

For Type A/B pancreatic cancer, the resectable rate reached $100 \%$ (22/22). However, one patient with Type $B$ accepted venous resection as normal pancreatic tissue was present within the tumor and around the portal vein, resulting in a resection rate without venous resection of 95\% for Type A/B (21/22). For Type C, the resectable rate was $89 \%(8 / 9)$, and $55 \%$ for resection without venous resection. For Type D, the resectable rate was $47 \%(7 / 15)$, but only $7 \%$ for resection without venous resection $(1 / 15)$.

Loyer's criteria [7] were the first attempt to stratify patients with vascular invasion to distinguish clearly unresectable cases from potentially resectable cases [15]. The researchers calculated the resection rate for the included patients, which had a certain clinical significance. However, this method is complex and relatively subjective, and failed to provide definite definitions of resectable and unresectable tumors [15]. Moreover, Loyer et al. only paid attention to imaging features, and did not consider intraoperative and pathological findings, since a pathologist was not asked to prepare histologic sections of the vascular wall in the early cases [7]. This may have limited the accuracy of this system. In addition, arterial and venous infiltrations were not differentiated. There is another limitation as well: Type $\mathrm{C}$ is described as a hypodense tumor with a point of contact forming a convexity against the vessel. However, if a tumor, which is densely fibrotic, simply impinges the venous wall, thus having a convex border or point of contact with the vein, it would be
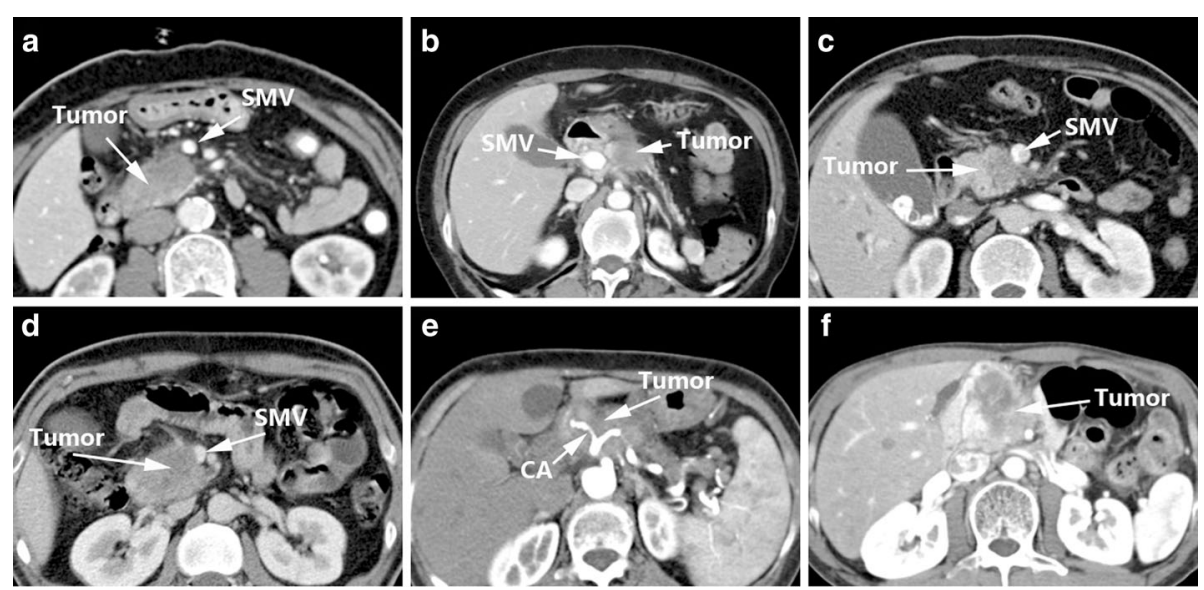

Fig. 1 Loyer's Criteria: Type A (a), Type B (b), Type C (c), Type D (d), Type E (e), Type F (f) 
Table 2 Lu's Criteria [12]

\begin{tabular}{ll}
\hline Grade & Imaging features \\
\hline 0 & No contiguity of tumor to vessel. \\
1 & Tumor contiguous to less than one-quarter circumference. \\
2 & Between one-quarter and one-half circumference. \\
3 & Between one-half and three-quarters circumference. \\
4 & Greater than three-quarters circumferential involvement \\
& or any vessel constriction. \\
\hline
\end{tabular}

classified as type C. However, this imaging finding was later-on called to be a "tear-drop deformity", which is actually highly suggestive of venous wall invasion.

\section{Lu's criteria (1997)}

In 1997, Lu et al. [12] assessed 25 patients who underwent surgery for pancreatic adenocarcinoma and designed classification criteria for tumor resectability (Table 2). Imaging features of peripancreatic vessels were the main assessment for this criteria, and were classified into five grades: Grade 0: the tumor does not touch adjacent vessels; Grade 1: less than one quarter of the tumor circumference contacts vessels; Grade 2: one quarter to half of the tumor circumference contacts vessels; Grade 3: half to three quarters of the tumor circumference contacts vessels; Grade 4: over three quarters of the tumor circumference contacts vessels, or any vascular constriction (Fig. 2). When combined with intra-operative assessment, the higher the grade, the lower the resectability rate.

Lu's criteria [12] considered a vessel circumferential involvement of $1 / 2\left(180^{\circ}\right)$ as the threshold of whether the tumor was resectable, which resulted in a sensitivity and specificity of 84 and $98 \%$, respectively, and a positive predictive value (PPV) and negative predictive value (NPV) for unresectability of 95 and 93\% (Table 7). These criteria were subsequently recognized and used by many scholars [16-18]. However, Lu's criteria only focused on circumferential involvement, and ignored other important parameters like the length of tumor contact and stenosis, which could explain their relatively low sensitivity (84\%). In addition, Valls et al. [15] stated that the main limitations of Lu's criteria [12] were that only 11 patients were eventually resectable, and most of the surgical correlations were based on venous vessels.

\section{Li's criteria (2005)}

$\mathrm{Li}$ et al. reported sequential studies [8, 9] in 2005 and 2006 and designed a set of criteria for arterial and venous invasion in pancreatic cancer according to imaging features and intra-operative findings (Table 3). The criteria could be divided into four signs: Sign A: arteries embedded within the tumor or blocked veins; Sign B: circumferential involvement greater than $180^{\circ}$; Sign C: irregular vessel walls; and Sign D: vessel caliber stenosis. Then two criteria were recommended. Criteria of arterial invasion: presence of sign $\mathrm{A}$, or combination of sign $\mathrm{B}$ with either sign $C$ and/or D. Criteria of venous invasion: presence of one of the following signs: sign $A$, sign $B$, sign $C$, sign D and sign $E$ (teardrop SMV) (Fig. 3).

The heterogeneity of Li's criteria $[8,9]$ is acceptable, but this system had a low sensitivity when used for assessment of artery and venous involvement. Therefore, the researchers realized specific assessments are needed for arterial and venous invasion (Nakayama et al. [19] expressed a similar opinion in 2001). In their recommended criteria, Li et al. [8, 9] stated artery invasion may meet Sign A or Sign B combined with either Sign C or Sign D, and venous invasion may meet Sign A, Sign B, Sign C, Sign D or Sign E (teardrop shape performance of the superior mesenteric vein). The sensitivity of these arterial and venous assessments for vessel invasion reached $79 \%(23 / 29)$ and $92 \%(45 / 49)$, respectively
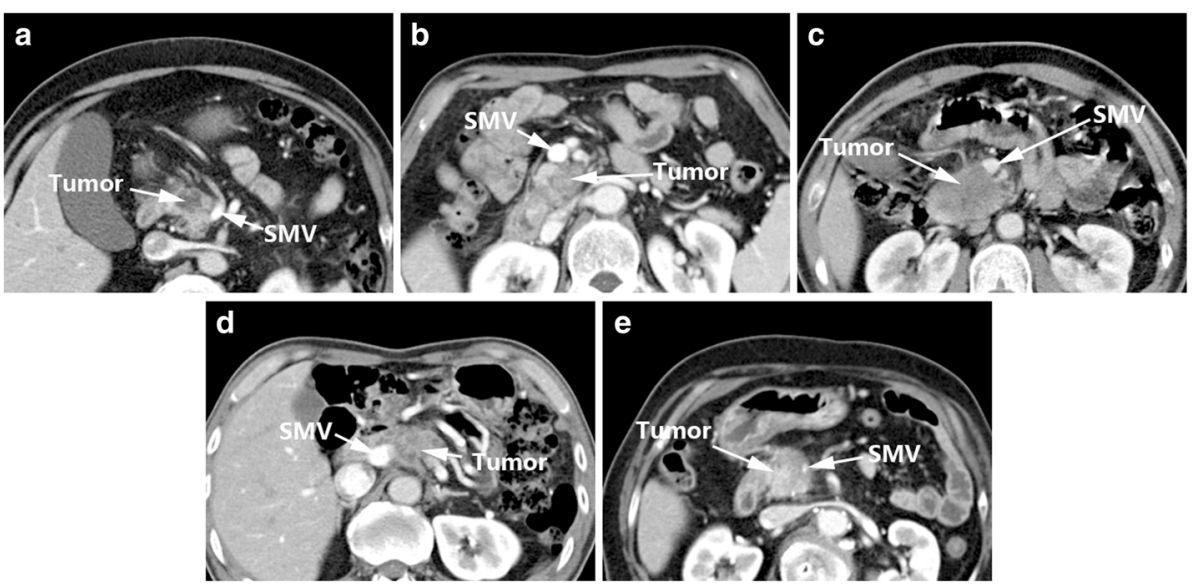

Fig. 2 Lu's Criteria: Grade 0 (a), Grade 1 (b), Grade 2 (c), Grade 3 (d), Grade 4 (e) 
Table 3 Li's Criteria $[8,9]$

\begin{tabular}{ll}
\hline Sign & Imaging features \\
\hline A & Arterial embedment in tumor or \\
& venous obliteration. \\
B & $\begin{array}{l}\text { Tumor surrounding } 1 / 2 \\
\text { circumference of the vessel. } \\
\text { C }\end{array}$ \\
D & Vessel wall irregularity. \\
\hline
\end{tabular}

Recommended criteria

Criteria of arterial invasion: presence of sign $\mathrm{A}$, or combination of sign B with either sign $C$ and/or D

Criteria of venous invasion: presence of one of the following signs: sign $A$, sign $B$, sign $C$, sign D and sign E (teardrop SMV)

(Table 7). The researchers considered that venous and arterial invasion present different $\mathrm{CT}$ signs of invasion, because the venous wall is thinner and weaker than the muscular arterial wall. When veins are surrounded or infiltrated by tumor, the wall tends to be irregular and the calibre becomes narrowed. At the same time, tumor often penetrates the venous wall and forms thrombus since the flow rate in veins becomes slow, causing venous occlusion finally [8].
Klauss's criteria (2008)

In 2008, Klauss et al. [13] proposed a new preoperative CT system to assess the resectability of pancreatic cancer (Table 4) based on the relation of peripancreatic vessels to the tumor, and verified the results using intraoperative findings and postoperative pathological reports. In this system, artery and venous assessments are separate (Fig. 4). Compared to the previous versions described above, Klauss's criteria include more assessment items and more detail. For example, the venous assessment includes assessment of the length of tumor contact, circumferential involvement and other abnormalities; the length of tumor contact and circumferential involvement are recorded to an accuracy of $\mathrm{mm}$ and degrees. The length of tumor contact and circumferential involvement assessment was also added for the artery assessment. Furthermore, this system provides a corresponding score for each assessment item, and the total score is calculated by adding the score for each item after the assessment. Generally, the total score was used to judge the resectability of the tumor and assess peripancreatic vessel invasion. Finally, 11 points was selected as the cut-off point for

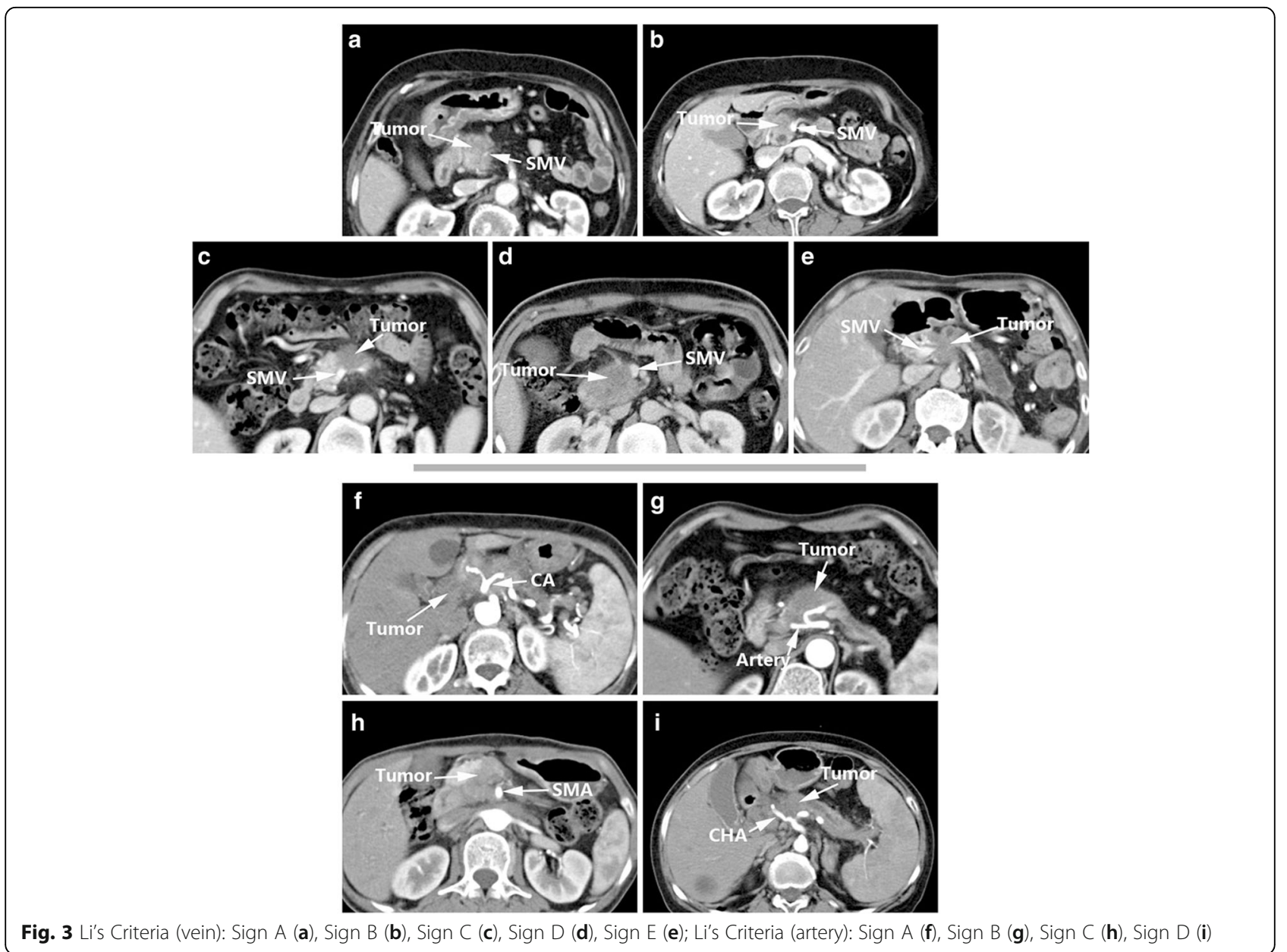


Table 4 Klauss's Criteria [13]

\begin{tabular}{|c|c|c|c|}
\hline $\begin{array}{l}\text { Length of tumor } \\
\text { contact }(\mathrm{mm})\end{array}$ & $\begin{array}{l}\text { Circumferential } \\
\text { Involvement }\left({ }^{\circ}\right)\end{array}$ & Other abnormalities & Score \\
\hline \multicolumn{4}{|l|}{ Veins } \\
\hline 0 & 0 & & 1 \\
\hline$<5$ & $1-45$ & & 2 \\
\hline $5-10$ & $46-90$ & & 3 \\
\hline $11-20$ & $91-180$ & Flattened & 4 \\
\hline $21-40$ & $181-270$ & $\begin{array}{l}\text { Long-segment } \\
\text { contour deformity }\end{array}$ & 5 \\
\hline$>40$ & $>270$ & $\begin{array}{l}\text { Obliteration or } \\
\text { severe contour } \\
\text { deformity }\end{array}$ & 6 \\
\hline Total score & & & $\Sigma$ \\
\hline \multicolumn{4}{|l|}{ Arteries } \\
\hline 0 & No & & 1 \\
\hline$<5$ & In Places & & 2 \\
\hline $5-10$ & Continuously $<45$ & & 3 \\
\hline $11-20$ & $45-180$ & & 4 \\
\hline $21-40$ & $181-270$ & & 5 \\
\hline$>40$ & $\begin{array}{l}270 \text { to complete } \\
\text { obliteration }\end{array}$ & & 6 \\
\hline Total score & & & $\Sigma$ \\
\hline
\end{tabular}

evidence of vessel invasion. That is to say, the vessel was invaded by the tumor in case of the total score of single vessel was equivalent with or higher than 11 points.

One major limitation of Klauss' Score is the fact that this very meticulous scoring system was developed with the same patient cohort, which was then also used to validate the score. For such an advanced scoring system a separate validation cohort would have been reliable. Based on their criteria, Klauss et al. [13] verified whether the superior mesenteric vein (SMV), superior mesenteric artery (SMA), splenic vein and portal vein (PV) or celiac trunks were involved in each patient. The sensitivity of this method for vessel invasion reached 66.7 to $100 \%$ (Table 7). Among the 28 patients, the sensitivity and specificity of the tumor resectability assessment reached 95.5\% (21/22) and $100 \%$ (6/6), respectively [13]. Compared to other related systems or criteria, Klauss's criteria have a higher sensitivity and specificity and warrant increased use in the clinic. However, Klauss et al. [13] stated their criteria also had a number of limitations, including the fact benign tumors would also lead to vessel compression and could lead to diagnostic errors.

\section{Marinelli's criteria (2014)}

The assessment system designed by Marinelli et al. [10] (Table 5) was mainly designed to assess peripancreatic venous invasion such as portal vein (PV) and superior mesenteric vein $(\mathrm{SMV})$, with the aim of selecting the appropriate therapeutic approach after accurate preoperative assessment to improve the treatment and prognosis of patients with borderline resectable disease. Compared to other criteria, the design of this system is more complicated. The items assessed are: tumor contact with vessel, length of tumor contact, circumferential involvement and stenosis. It is noteworthy that the tumor contact with vessel criterion employed the system included in Loyer's criteria [7]. However, interestingly Marinelli et al. [10] combined Loyer's Grade A and Grade B in their system. Marinelli et al. maintained there is no significant difference between these two grades in terms of surgical outcome, since both
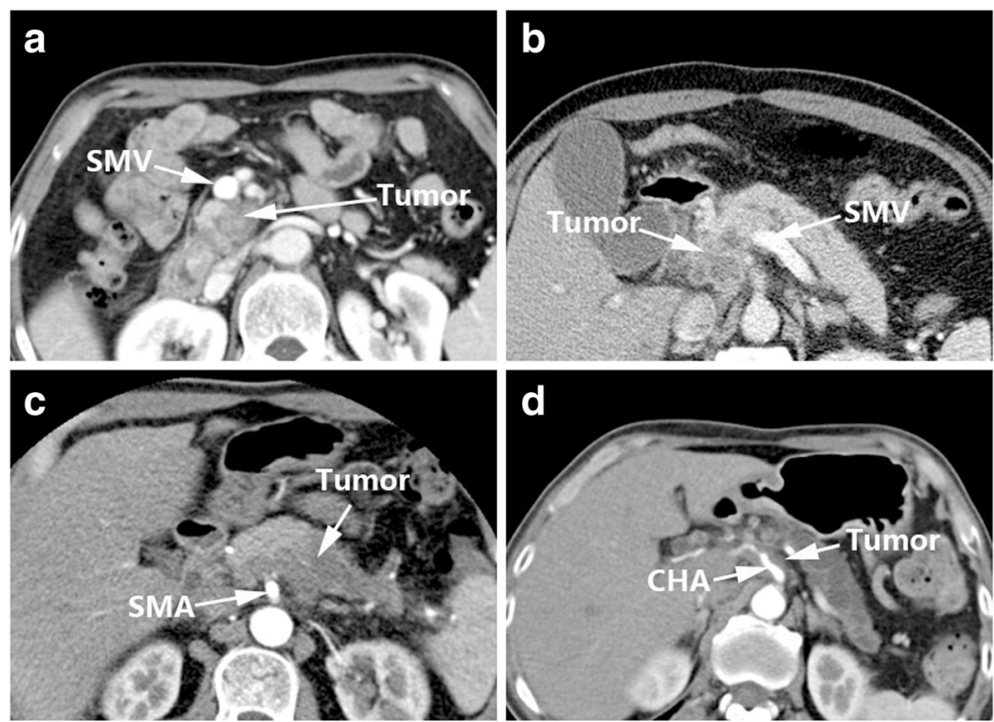

Fig. 4 Klauss's Criteria (vein): Score $<11$ (a), Score $>11$ (b); Klauss's Criteria (artery): Score $<11$ (c), Score $>11$ (d) 
Table 5 Marinelli's Criteria [10]

\begin{tabular}{lllll}
\hline $\begin{array}{l}\text { Grade } \\
\text { (likelihood } \\
\text { of vascular } \\
\text { invasion) }\end{array}$ & $\begin{array}{l}\text { Tumor } \\
\text { with vessel }\end{array}$ & $\begin{array}{l}\text { Length of } \\
\text { tumor contact } \\
\text { with vessel } \\
(\mathrm{mm})\end{array}$ & $\begin{array}{l}\text { Circumferential } \\
\text { vein } \\
\text { involvement }\left(^{\circ}\right)\end{array}$ & Stenosis \\
\hline 1 & Grade A-B & $=0 \mathrm{~mm}$ & $=0^{\circ}$ & No stenosis \\
2 & Grade C & $<5 \mathrm{~mm}$ & $=0^{\circ}-90^{\circ}$ & No stenosis \\
3 & Grade C-D & $>5 \mathrm{~mm}$ & $=0^{\circ}-90^{\circ}$ & Flattened \\
4 & Grade D & $>5 \mathrm{~mm}$ & $>90^{\circ}<180^{\circ}$ & $\begin{array}{l}\text { Occlusion } \\
\text { thrombus }\end{array}$ \\
& & & $>180^{\circ}$ & - \\
\hline
\end{tabular}

Grade 1, Definite absence of invasion; Grade 2, Probable absence of invasion; Grade 3, Probable presence of invasion; Grade 4, Definite presence of invasion ${ }^{a}$ Grades A-F, according to Loyer's Criteria [8]:

Grade A: fat plane visible between tumour and vessels

Grade B: normal pancreatic tissue between tumour and vessels

Grade C: tumour adjacent to vessel with a convex contour towards vessels

Grade D: tumour adjacent to vessel with a concave contour towards vessels

Grade E: circumferential involvement of vessels

Grade F: vascular occlusion

are clearly resectable [10]. The "length of tumor contact" was classified as $0 \mathrm{~mm},<5 \mathrm{~mm}$ and $>5 \mathrm{~mm}$; "circumferential involvement" as $0^{\circ}, 0^{\circ}$ to $90^{\circ}, 90^{\circ}$ to $180^{\circ}$, and $>180^{\circ}$, respectively. Four grades were defined in Marinelli's criteria [10]: Grade 1, definite absence of invasion; Grade 2, probable absence of invasion; Grade 3, probable presence of invasion; and Grade 4, definite presence of invasion. In Grade 1 , the tumor contacts with vessels of Grade A-B, and length of tumor contact is $0 \mathrm{~mm}$ with circumferential involvement of 0 and no stenosis. In Grade 2, the tumor contacts with vessels of Grade $\mathrm{C}$, with a length of tumor contact $<5 \mathrm{~mm}$, circumferential involvement is $0^{\circ}-90^{\circ}$ and no stenosis. There are two kinds of situations in Grade 3, the tumor contacts with vessels of Grade $C$, with a length of tumor contact $>5 \mathrm{~mm}$, circumferential involvement is $0^{\circ}-90^{\circ}$ or flattened vessels. Another situation is circumferential involvement is $0-90^{\circ}$ with flattened vessels, Grade D tumor vessel contact. Grade 4 includes three scenarios: grade $\mathrm{E}$ or $\mathrm{F}$ tumor vessel contact and circumferential involvement $>180^{\circ}$; narrowing of vessels; or Grade D tumor contact, contact length $>5 \mathrm{~mm}$ and circumferential involvement of $90^{\circ}$ to $180^{\circ}$ (Fig. 5).

The advantage of Marinelli's score over Klauss' criteria [13] is the fact that Marinelli's scoring system refers to actual clinical situations instead of adding score numbers. Marinelli et al. [10] verified their standard in 56 patients with pancreatic cancer and obtained sensitivity and specificity values for PV invasion of 80 and 100\%, respectively. The PPV and NPV were 80 and 96\%. For the SMV, the sensitivity and specificity of this method reached 100 and $94 \%$, and the PPV and NPV were 75 and 100\% (Table 7). The innovation in this method was that the researchers analyzed the prognosis of the patients by grade. For the PV infiltration score, the survival time was inversely proportional to grade, though the trend was not significant $(P=0.106)$. Additionally, the researchers proposed that both the PV and SMV infiltration scores were associated with metastatic disease and the resection margins status [10].

\section{Teramura's criteria (2016)}

Teramura et al. [11] assessed whether pathological PV invasion (pPV) in pancreatic cancer could be accurately identified by preoperative $\mathrm{CT}$ in order to select patients who could benefit from surgery. The researchers established a CT diagnostic standard according to the degree of vascular invasion, intra-operative findings and pathology results (Table 6). The classification method for this criteria is similar to Loyer's criteria [7] and is divided into five types (Type
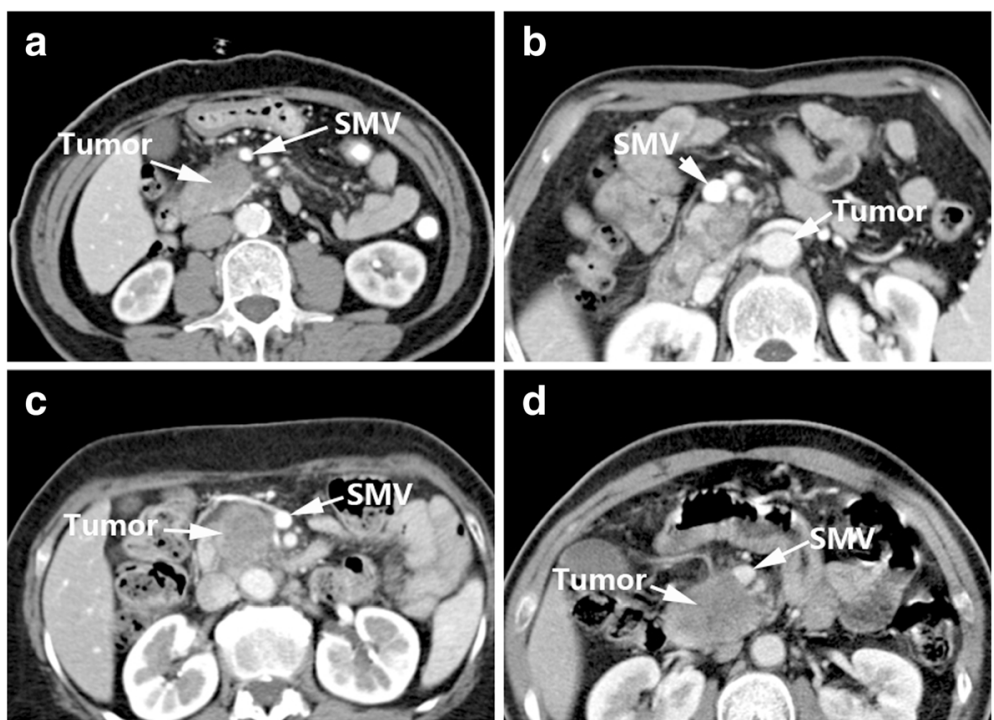

Fig. 5 Marinelli's Criteria: Grade 1 (a), Grade 2 (b), Grade 3 (c), Grade 4 (d) 
Table 6 Teramura's Criteria [11]

\begin{tabular}{llll}
\hline Type & Diagnosis & CT findings & \\
\hline 0 & Negative & Negative & c vessels. \\
1 & Soft tissue density & $\begin{array}{l}\text { Soft tissue density between } \\
\text { tumor and portal vein. }\end{array}$ \\
2 & Positive & Contact & $\begin{array}{l}\text { Tumor is inseparable from } \\
\text { adjacent vessels, and points } \\
\text { of contact from a convexity } \\
\text { against the vessels. }\end{array}$ \\
& & Deformation, narrowing or \\
3 & Stenosis & $\begin{array}{l}\text { Stenosis on portal vein. } \\
\text { Portal vein is completely } \\
\text { obstructed by tumor. }\end{array}$ \\
\hline
\end{tabular}

0 - 4): In Type 0, a fat plane separates the tumor and (or) normal pancreatic tissues from adjacent vessels; in Type 1, soft tissue density exists between the tumor and vessels; in Type 2, the tumor cannot be separated from the adjacent vessels and the points of contact from a convexity against the vessels; in Type 3, the PV is deformed, narrowed or exhibits stenosis; and in Type 4, the PV is completely blocked by the tumor (Fig. 6).

Teramura et al. [11] demonstrated that the prognosis of Type 0 vs. Type $3 / 4$ was significantly different $(P=0.02)$, but not for Types 0 vs. $1 / 2$ and Types $1 / 2$ vs. $3 / 4(P=0.30$ and $P=0.10$, respectively). The 5 -years survival rates for Type $0,1 / 2$ and $3 / 4$ were $23.1,11.4$ and $3.2 \%$, respectively. Although a significant difference in 5-years survival was not observed between Type $1 / 2$ and Type 3/4, a higher percentage of patients with Type $1 / 2$ than Type $3 / 4$ survived for 36 months (10/35 vs. 1/32). Therefore, this method was feasible to assess whether patients are suitable for pancreaticoduodenectomy with PV resection via preoperative CT [11]. However, while the sensitivity and NPV were 97.6 and $97.5 \%$, respectively, the specificity was only $60 \%$ and the PPV was $61.2 \%$ (Table 7 ).

\section{Clinical significance}

With respect to resectability, patients with Type A and B vascular involvement according to Loyer's criteria [7] are suggested to undergo pancreatic resection, while Type E and $\mathrm{F}$ are considered inoperable. In their study, one case of Type E/F underwent surgery with vessel resection, though a positive margin was detected in the pathological examination. Resection was recommended for Type C, but the tumor may or may not attach to the vessel wall. A detailed plan of the surgical approach should be made before pancreatic resection in cases of Type D. It is important to note that venous resection should not be attempted if the surgeon lacks relevant experience. In addition, Loyer's [7] study did not provide a definite definition of resectable and unresectable, as previously discussed. Teramura et al. [11] mainly focused on the relationship between prognosis and vascular invasion. They reported patients with "stenosis", "obstruction", or a Klauss score $[13] \geq 11$ are likely to have a poor prognosis, even with portal vein reconstruction (PVR) [11], and recommended resectability should be assessed from the perspective of prognosis. According to the aforementioned data, $\mathrm{Lu}$ et al. [12] used one-half of the circumference of the vessel as the threshold; resection should be recommended if the value was higher. Furthermore, Li et al. $[8,9]$ and Hough et al. [20] found that a tear drop appearance of the SMV can be a contraindication for resection. However, unambiguous definitions of resectable tumors were not provided in the criteria by Klauss [13] and Marinelli [10].

In recent years, these rigid concepts of vascular invasion (meaning non-resectability) have been somehow overruled by the concept of "borderline resectable", which has been adopted by many cancer centers and institutions. According to the NCCN guidelines (Version 1.2017), the "borderline resectable" could be defined as several resectability statuses as follows:

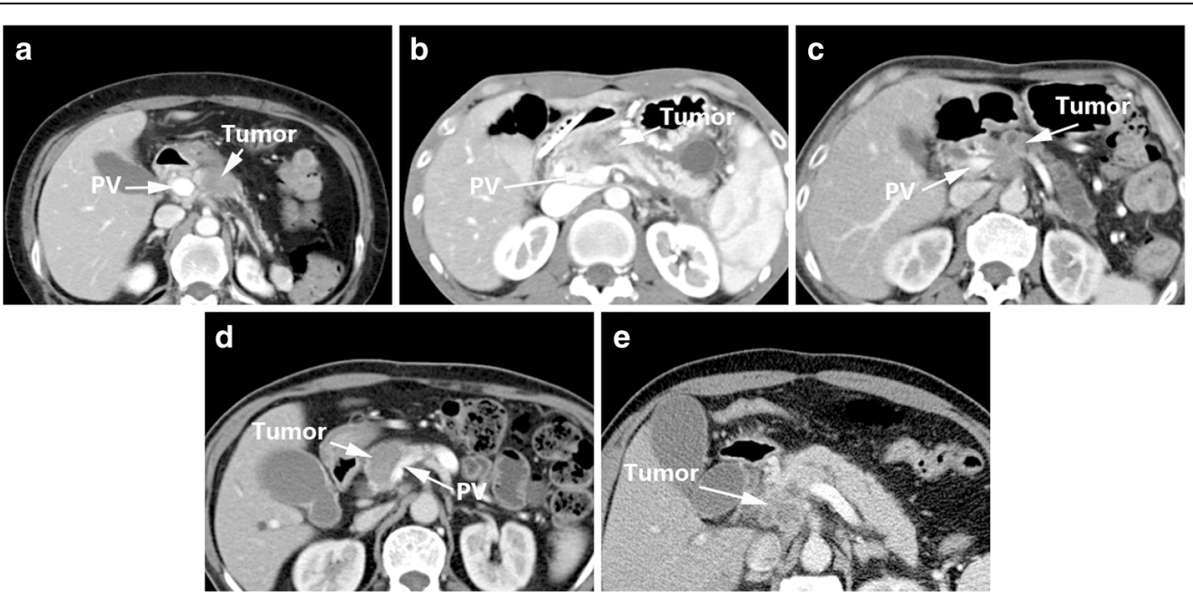

Fig. 6 Teramura's Criteria: Type 0 (a), Type 1 (b), Type 2 (c), Type 3 (d), Type 4 (e) 
Table 7 Sensitivity and specificity of each criteria for vessel invasion in pancreatic cancer

\begin{tabular}{|c|c|c|c|c|c|}
\hline Criteria & Vessel & Sensitivity (\%) & Specificity (\%) & PPV (\%) & NPV (\%) \\
\hline $\begin{array}{l}\text { Loyer et al. } \\
(n=56)\end{array}$ & NA & NA & NA & NA & NA \\
\hline $\begin{array}{l}\text { Lu et al. } \\
(n=25)\end{array}$ & $\begin{array}{l}\text { Vein/ } \\
\text { Artery }\end{array}$ & 84 & 98 & 95 & 93 \\
\hline \multirow{2}{*}{$\begin{array}{l}\text { Li et al. } \\
(n=54)\end{array}$} & Vein & 92 & 100 & NA & NA \\
\hline & Artery & 79 & 99 & NA & NA \\
\hline \multirow{5}{*}{$\begin{array}{l}\text { Klauss et al. } \\
(n=28)\end{array}$} & SMV & 100 & 95.8 & 80 & 100 \\
\hline & $\begin{array}{l}\text { Splenic } \\
\text { vein }\end{array}$ & 66.7 & 100 & 100 & 96.2 \\
\hline & PV & 100 & 96.2 & 66.7 & 100 \\
\hline & $\begin{array}{l}\text { Celiac } \\
\text { trunk }\end{array}$ & 100 & 100 & 100 & 96.4 \\
\hline & SMA & 100 & 100 & 100 & 96.4 \\
\hline \multirow{2}{*}{$\begin{array}{l}\text { Marinelli } \\
\text { et al. } \\
(n=56)\end{array}$} & PV & 80 & 100 & 80 & 96 \\
\hline & SMV & 96 & 94 & 75 & 100 \\
\hline $\begin{array}{l}\text { Teramura } \\
\text { et al. } \\
(n=107)\end{array}$ & PV/SMV & 97.6 & 60 & 61.2 & 97.5 \\
\hline
\end{tabular}

$P V$ portal vein, SMV superior mesenteric vein, SMA superior mesenteric artery, NA not available, PPV positive predictive value, NPV negative predictive value

1. Venous

- Solid tumor contact with SMV or PV of $>180^{\circ}$, contact of $<=180^{\circ}$ with contour irregularity of the vein or thrombosis of the vein but with suitable vessel proximal and distal to the site of involvement allowing for safe and complete resection and vein reconstruction;

- Solid tumor contact with the inferior vena cava (IVC).

2. Arterial

2.1Pancreatic head/uncinate process:

- Solid tumor contact with common hepatic artery (CHA) without extension to celiac axis or hepatic artery bifurcation allowing for safe and complete resection and reconstruction;

- Solid tumor contact with the superior mesenteric artery (SMA) of $<=180^{\circ}$;

- Solid tumor contact with variant arterial anatomy (ex: accessory right hepatic artery, replaced right hepatic artery, replaced $\mathrm{CHA}$, and the origin of replaced or accessory artery) and the presence and degree of tumor contact should be noted if present as it may affect surgical planning.

2.2Pancreatic body/tail:

- Solid tumor contact with the celiac axis (CA) of $<=180^{\circ}$;

- Solid tumor contact with the CA of $>180^{\circ}$ without involvement of the aorta and with intact and uninvolved gastroduodenal artery thereby permitting a modified Appleby procedure.

Interestingly, Teramura et al. [11] doubted the definition of "borderline" pancreatic head cancer established in the newest NCCN guidelines, and pointed out that circumferential contact of the PV did not have high diagnostic value and may even affect assessment of the resectability of "borderline" pancreatic head cancer.

Other studies also considered prognosis. Nakao et al. [21] showed the imaging features of PV correlated with long-term survival; survival was poorer for patients with bilateral narrowing or stenosis/obstruction with collaterals than patients with unilateral narrowing [21]. Moreover, they also suggested that radiographic classification of PV invasion was more appropriate than pathological classification [21]. A similar report by Chun et al. [22] showed patients with bilateral narrowing were less likely to benefit from preoperative treatment.

Another useful feature of these criteria [7-13] summarized by us is the prediction of vascular invasion. Some researchers previously believed perivascular changes were not specific for pancreatic carcinoma [23-25]. However, Megibow maintained that patients with pathological confirmed ductal adenocarcinoma are likely to have tumor infiltration if perivascular changes can be observed on CT [26]; this supposition was supported by Loyer [7]. In the study by Zeman et al. [27], vascular invasion could be identified if the caliber was irregular, circumferential involvement $>180^{\circ}$, or vessel thrombosis was present. In the study by Furukawa et al. [28], vascular invasion is classified as positive if circumferential involvement is more than $90^{\circ}$. Klauss et al. [13] considered it was difficult to assess vascular invasion as the contact between the vessels and tumor does not always indicate whether the vessels have been truly infiltrated; Teramura et al. [11] expressed a similar view. In research published in 2012, Nakao et al. [21] found imaging classifications of PV invasion correlated with the pathological grade of invasion.

\section{Conclusions}

From the information above, we can conclude that the previous studies suggesting criteria for assessing the resectability of pancreatic cancer via CT are basically consistent, and while some criteria are suitable for clinical practice (for example, the sensitivity and specificity of the Klauss's criteria [13] reach 95.5 and $100 \%$, respectively), they also remain controversial. In most studies, the length of tumor contact, circumferential involvement, stenosis and other imaging findings are taken as reference items. However, in the latest study, Teramura et al. [11] reported circumferential involvement had low diagnostic value and they removed this feature from their criteria. In addition, 
scoring systems with a high reference value like Klauss's criteria also have limitations. For instance, it is difficult to distinguish whether vessels are oppressed by a benign or malignant tumor on CT.

In conclusion, we hold the opinion that the current criteria [7-13] have superior clinical value to previous systems, The scoring system, especially from Klauss' and Marinelli's, is worthy of being applied to the clinical practice. However, the criteria above still remain controversial, especially with respect to the lack of the prognostic criteria. We believe that with continuous developments in CT technology and accumulation of experience by radiologists, more improved and accurate criteria will be established.

\section{Abbreviations \\ CA: Celiac axis; CHA: Hepatic artery; CT: Computed tomography; NCCN: National comprehensive cancer network; NPV: Negative predictive value; PPV: Positive predictive value; PV: Portal vein; SMA: Superior mesenteric artery; SMV: Superior mesenteric vein}

\section{Acknowledgements}

Not applicable.

\section{Funding}

This study is supported by National High Technology Research and Development Program of China (No. SS2015AA020405), Training Program of the Key Program of the National Natural Science Foundation of China (No. 91442115), National Natural Science Foundation of China (No.81672337), Key Program of the National Natural Science Foundation of China (No. 81530079), Key research and development Project of Zhejiang Province (No. 2015C03044), Zhejiang Provincial Program for the Cultivation of High-level Innovative Health talents, Zhejiang Provincial Key Innovation Team of Pancreatic Cancer Diagnosis \& Treatment (No.2013TD06).

\section{Availability of data and materials}

Not applicable.

\section{Authors' contributions}

All the authors contributed equally to this work. All authors read and approved the final manuscript.

\section{Competing interests}

The authors who have taken part in this study declared that they do not have any conflict of interest with respect to this manuscript.

\section{Consent for publication}

All the authors have consented for publication of this manuscript.

\section{Ethics approval and consent to participate}

Not applicable.

\section{Publisher's Note}

Springer Nature remains neutral with regard to jurisdictional claims in published maps and institutional affiliations.

Received: 7 December 2016 Accepted: 27 April 2017

Published online: 06 May 2017

\section{References}

1. Kamisawa T, Wood LD, Itoi T, Takaori K. Pancreatic cancer. Lancet. 2016; 388(10039):73-85.

2. Siegel RL, Miller KD, Jemal A. Cancer statistics, 2016. CA Cancer J Clin. 2016; 66(1):7-30.

3. Ryan DP, Hong TS, Bardeesy N. Pancreatic adenocarcinoma. N Engl J Med. 2014;371(22):2140-1.

4. Gillen S, Schuster T, Meyer ZBC, Friess H, Kleeff J. Preoperative/neoadjuvant therapy in pancreatic cancer: a systematic review and meta-analysis of response and resection percentages. PLoS Med. 2010;7(4):e1000267.
5. White R, Winston C, Gonen M, et al. Current utility of staging laparoscopy for pancreatic and peripancreatic neoplasms. J Am Coll Surg. 2008;206(3):445-50.

6. Pietryga JA, Morgan DE. Imaging preoperatively for pancreatic adenocarcinoma. J Gastrointest Oncol. 2015;6(4):343-57.

7. Loyer EM, David CL, Dubrow RA, Evans DB, Charnsangavej C. Vascular involvement in pancreatic adenocarcinoma: reassessment by thin-section CT. Abdom Imaging. 1996;21(3):202-6.

8. Li H, Zeng MS, Zhou KR, Jin DY, Lou WH. Pancreatic adenocarcinoma: the different $C$ criteria for peripancreatic major arterial and venous invasion. J Comput Assist Tomogr. 2005;29(2):170-5.

9. Li H, Zeng MS, Zhou KR, Jin DY, Lou WH. Pancreatic adenocarcinoma: signs of vascular invasion determined by multi-detector row CT. Br J Radiol. 2006; 79(947):880-7.

10. Marinelli T, Filippone A, Tavano F, et al. A tumour score with multidetector spiral $C T$ for venous infiltration in pancreatic cancer: influence on borderline resectable. Radiol Med. 2014;119(5):334-42.

11. Teramura K, Noji T, Nakamura T, et al. Preoperative diagnosis of portal vein invasion in pancreatic head cancer: appropriate indications for concomitant portal vein resection. J Hepatobiliary Pancreat Sci. 2016;23(10): 643-9.

12. Lu DS, Reber HA, Krasny RM, Kadell BM, Sayre J. Local staging of pancreatic cancer: criteria for unresectability of major vessels as revealed by pancreaticphase, thin-section helical CT. AJR Am J Roentgenol. 1997;168(6):1439-43.

13. Klauss $\mathrm{M}$, Mohr A, von Tengg-Koblig H, et al. A new invasion score for determining the resectability of pancreatic carcinomas with contrast-enhanced multidetector computed tomography. Pancreatology. 2008;8(2):204-10.

14. Tempero MA, Malafa MP, Behrman SW, et al. Pancreatic adenocarcinoma, version 2.2014: featured updates to the NCCN guidelines. J Natl Compr Canc Netw. 2014;12(8):1083-93.

15. Valls C, Andía E, Sanchez A, et al. Dual-phase helical CT of pancreatic adenocarcinoma: assessment of resectability before surgery. AJR Am J Roentgenol. 2002;178(4):821-6.

16. Horton KM, Fishman EK. Multidetector CT angiography of pancreatic carcinoma: part 2, evaluation of venous involvement. AJR Am J Roentgenol. 2002;178(4):833-6.

17. Lepanto L, Arzoumanian Y, Gianfelice D, et al. Helical CT with CT angiography in assessing periampullary neoplasms: identification of vascular invasion. Radiology. 2002;222(2):347-52.

18. O'Malley ME, Boland GW, Wood BJ, Fernandez-del CC, Warshaw AL, Mueller PR. Adenocarcinoma of the head of the pancreas: determination of surgical unresectability with thin-section pancreatic-phase helical CT. AJR Am J Roentgenol. 1999;173(6):1513-8.

19. Nakayama Y, Yamashita Y, Kadota M, et al. Vascular encasement by pancreatic cancer: correlation of $C T$ findings with surgical and pathologic results. J Comput Assist Tomogr. 2001;25(3):337-42.

20. Hough TJ, Raptopoulos V, Siewert B, Matthews JB. Teardrop superior mesenteric vein: $C T$ sign for unresectable carcinoma of the pancreas. AJR Am J Roentgenol. 1999;173(6):1509-12.

21. Nakao A, Kanzaki A, Fujii T, et al. Correlation between radiographic classification and pathological grade of portal vein wall invasion in pancreatic head cancer. Ann Surg. 2012;255(1):103-8.

22. Chun YS, Milestone BN, Watson JC, et al. Defining venous involvement in borderline resectable pancreatic cancer. Ann Surg Oncol. 2010;17(11):2832-8,

23. Schulte SJ, Baron RL, Freeny PC, Patten RM, Gorell HA, Maclin ML. Root of the superior mesenteric artery in pancreatitis and pancreatic carcinoma: evaluation with CT. Radiology. 1991;180(3):659-62.

24. Luetmer PH, Stephens DH, Fischer AP. Obliteration of periarterial retropancreatic fat on $\mathrm{CT}$ in pancreatitis: an exception to the rule. AJR Am J Roentgenol. 1989;153(1):63-4.

25. Baker ME, Cohan RH, Nadel SN, Leder RA, Dunnick NR. Obliteration of the fat surrounding the celiac axis and superior mesenteric artery is not a specific CT finding of carcinoma of the pancreas. AJR Am J Roentgenol. 1990;155(5):991-4

26. Megibow AJ. Pancreatic adenocarcinoma: designing the examination to evaluate the clinical questions. Radiology. 1992;183(2):297-303.

27. Zeman RK, Cooper C, Zeiberg AS, et al. TNM staging of pancreatic carcinoma using helical CT. AJR Am J Roentgenol. 1997;169(2):459-64.

28. Furukawa $H$, Kosuge $T$, Mukai $K$, et al. Helical computed tomography in the diagnosis of portal vein invasion by pancreatic head carcinoma: usefulness for selecting surgical procedures and predicting the outcome. Arch Surg. 1998;133(1):61-5. 